1. FCPS (Dermatology) Assistant Professor

DUHS Civil Hospital Karachi.

2. FCPS (Dermatology)

Assistant Professor

DUHS Civil Hospital Karachi.

3. FCPS (Dermatology)

Assistant Professor

DOW International Medical College.

4. FCPS (Dermatology) Assistant Professor DUHS Civil Hospital Karachi.

5. FCPS (Dermatology)

Professor of Dermatology CIVIL Hospital Karachi.

6. MBBS

PG Trainee Dermatology

Civil Hospital Karachi.

Correspondence Address:

Dr. Humaira Talat

Assistant Professor

DUHS Civil Hospital Karachi.

hmrtalat@yahoo.com

Article received on:

09/01/2017

Accepted for publication:

30/04/2017

Received after proof reading: 03/07/2017

\section{PEMPHIGUS VULGARIS;}

FREQUENCY OF PULMONARY TUBERCULOSIS IN PATIENTS ON IMMUNOSUPPRESSIVE DRUGS

Humaira Talat ${ }^{1}$, Reema Mirza², Madiha Sajid ${ }^{3}$, Maria Mansoor ${ }^{4}$, Zarnaz Wahid $^{5}$, Saba Majeed ${ }^{6}$

ABSTRACT... Objectives: To determine the frequency of Pulmonary Tuberculosis in Pemphigus Vulgaris patients on immunosuppressive therapy for more than one year. Study Design: Prospective Cross Sectional. Plan and Duration of Study: The study was conducted at Dermatology department Civil Hospital Karachi (a tertiary care hospital affiliated with Dow University of Health Sciences). It was conducted from January 2016 to June 2016. Material and Methods: The study included 31 diagnosed cases of Pemphigus Vulgaris patients on immunosuppressive drugs for more than one year. These patients were either admitted in dermatology ward CHK or presented in CHK OPD. A Pro-forma was used to record the history, physical examination and results of screening tests. Results: Out of 31 Pemphigus Vulgaris patients who were on immunosuppressive drugs for more 1 year, 3 patients $(9.6 \%)$ developed Pulmonary Tuberculosis. Conclusion: Patients of Pemphigus Vulgaris on long term immunosuppressant drugs can develop Pulmonary Tuberculosis.

Key words: $\quad$ Pulmonary Tuberculosis, Pemphigus Vulgaris

Article Citation: Talat H, Mirza R, Sajid M, Mansoor M, Wahid Z, Majeed S. Pemphigus vulgaris; frequency of pulmonary tuberculosis in patients on immunosuppressive drugs. Professional Med J 2017;24(7):1081-1083. DOI: 10.17957/TPMJ/17.3814

\section{INTRODUCTION}

Tuberculosis is a mycobacterial infection caused by Mycobacterium Tuberculosis. It is an acid fast bacillus. Tuberculosis is a second leading cause of death from an infectious disease (after HIV infection). ${ }^{1}$ Tuberculosis affects various organs of the body including lungs, Gl tract, skin, bones, joints etc. The risk of acquiring tuberculosis is increased in immunocompromised individuals.

Pemphigus Vulgaris is an immunobullous disorder characterized by antibody mediated autoimmune response against structural elements of skin. This results in blistering of skin and mucosa. Antibody targets in Pemphigus Vulgaris include proteins in desmosomes. Therapeutic options include immunosuppressants eg. Corticosteroids, Azathioprin, Mycophenolates etc., immune modulators eg. Dapsone, Tetracyclines etc. Prolonged and high dose of immunosuppressants eg. Glucocorticoids, Azathioprin etc. are often required to control Pemphigus Vulgaris. Such immunocompromised patients are at risk of acquiring Primary Tuberculosis and reactivation of non-active Tuberculosis.

\section{MATERIAL AND METHODS}

This is a Prospective cross sectional study. It was conducted in Civil Hospital Karachi (a tertiary care hospital).The time period was from $1 / 1 / 16$ to $30 / 6 / 16$. The study included 31 diagnosed cases of Pemphigus Vulgaris on immunosuppressive treatment for more than one year. These patients were either admitted in dermatology ward Civil hospital Karachi or presented in CHK OPD.

\section{Inclusion Criteria}

1. Patients of both genders

2. All were diagnosed cases of Pemphigus Vulgaris. Diagnosis was made clinically and histologically by a dermatologist.

3. Diagnosed cases of Pemphigus Vulgaris receiving immunosuppressive for more than 1 year.

4. Age range $22-70$ years

\section{Exclusion Criteria}

1. Paediatric age group 
2. Other immunocompromised individuals eg. Transplant patients, HIV patients etc.

3. Patients on immunosuppressive therapy for less than 1 year

A pro-forma was used to record history, physical examination and results of screening tests

Following investigations were done

Routine Investigations Performed in All Patients

- Compete blood count

- ESR

- Urine detailed report

- Serum urea/creatinine /electrolytes

- Random blood Sugar

- X-ray Chest

\section{Specific Screening Test}

- Sputum

- Smear for AFB

- Culture for AFB

- X-ray Chest

- Montoux test

\section{RESULTS}

The study comprised of 31 patients. These patients were diagnosed cases of Pemphigus Vulgaris on immunosuppressive drugs Prednisolone and Aziathioprin for more than 1 year.

Out of these 31 patients - 12 (38.7\%) were male (age range- 22-70years) and 19(1.29\%) were female (age range 32-55 years).

Thestudy concluded that out ofatotal of 31 patients, $3(9.6 \%)$ suffering from Pemphigus Vulgaris, who were on Prednisolone and Azathioprin for more than 1 year developed pulmonary tuberculosis. All of these 3 patients were females. 1 patients (35 years) was suffering from Pemphigus Vulgaris since 10 years and receiving Prednisolone and Azathioprin. She developed pulmonary T.B after 7 years of receiving immunosuppressants. Other 2 patients (32 and 30 years) were suffering from Pemphigus Vulgaris since 18 months and receiving Prednisolone and Azathioprin for the disease. They developed pulmonary T.B. after 1 year of receiving these immunosuppressants.
All of these 3 patients of Pemphigus Vulgaris with pulmonary T.B were already treated with Anti Tuberculosis Treatment on presentation in CHK. Their M.T and sputum smear for AFB and X-ray chest were clear. However, previous record of M.T positive, sputum smear and culture for AFB positive and positive findings for T.B on X-ray chest were available.

All of these female Pemphigus Vulgaris patients who developed pulmonary Tuberculosis were married and housewives. They belonged to a poor socioeconomic class. There was no family history of Tuberculosis in any patient.

\section{DISCUSSION}

The primary objective of the therapeutic management of $P V$ is initially to control the disease, heal the bullous skin and mucous lesions, and minimize the associated functional impairment. Subsequently, the real challenge is to prevent relapses in the long run and avoid adverse events associated with the prolonged use of steroids and immunosuppressive agents. ${ }^{2}$ There are limited studies regarding the role of immunosuppressive drugs in precipitating tuberculosis. Some studies have shown that immunosuppressive therapy has increased the risk of developing tuberculosis while others conclude that there is negligible influence on the incidence of tuberculosis secondary to immunosuppressive therapy. ${ }^{3}$

In this study we found that 3 patients out of 31 patients developed tuberculosis after being treated with prednisolone and azathioprine for more than 1 year.

Kim et al. ${ }^{4}$ reported an increase in the incidence of active tuberculosis among 269 patients of rheumatoid disease on moderate to high doses of corticosteroids. The risk factors were, cumulative and mean daily steroid doses taken during the first year of therapy. Jick et al. ${ }^{5}$ found that patients treated with glucocorticoids have an increased risk of developing tuberculosis independent of other risk factors. In another study conducted on patients of dermatomyositis taking steroids and azathioprine that increase risk of developing T.B. ${ }^{6}$ 
Previous reports have demonstrated an association between several immune-mediated diseases and a subsequent risk of acquiring TB. ${ }^{6,7,8,9}$ In these studies, possible mechanisms that may have led to increased TB risk includes the immune dysfunction that results from immune-mediated diseases and the effects of the immunosuppressant drugs that are used to treat them. ${ }^{10}$

We can say that not only pemphigus vulgaris patients can develop tuberculosis if taking immunosuppressive therapy for longer period but it has been noticed in patients of Systemic lupus erythematosis and other rheumatological disorders on immunosuppressive therapy. ${ }^{2,3,11}$

\section{CONCLUSION}

Patients on long term immunosuppressive therapy eg. steroids, azathioprine, cyclosporin etc. are more vulnerable to develop various infections like Pulmonary Tuberculosis. Hence they should be screened for pulmonary tuberculosis by simple screening test like X-ray Chest, Sputum for AFB and Montoux test.

\section{Copyright(C) 30 Apr, 2017.}

\section{REFERENCES}

1. Raviglione MC, O’Brien RJ. Tuberculosis. In: Kasper DL, Braunwald E, Fauci AS, et al. Harrison's Principles of Internal Medicine, 16 thed. New York: McGraw-Hill; 2005. P.953-66.

2. Sayarlioglu M, Inanc M, Kamali Set al. Tuberculosis in Turkish patients with systemic lupus erythematosus: increased frequency of extrapulmonary localization. Lupus. 2004; 13:274-8.
3. Pal D, Behera D, Gupta D, Agarwal A. Tuberculosis in patients receiving prolonged treatment with oral corticosteroids for respiratory disorders. Indian $\mathrm{J}$ Tuberc. 2002; 49: 83.

4. $\mathrm{Kim} \mathrm{H}$, Yoo C, Baek J et al. Mycobacterium tuberculosis infection in a corticosteroid-treated rheumatic disease patient population. Clin Exp Rheumatol. 1998; 16:9-13.

5. Jick SS, Lieberman ES, Rahman MU, Choi HK. Glucocorticoid use, other associated factors, and the risk of tuberculosis. Arthritis Rheum. 2006; 55:1926.

6. Aberer W, Wolff-Schreiner EC, Stingl G, Wolff K. Azathioprine in the treatment of pemphigus vulgaris. J Am Acad Dermatol. 1987 Mar; 16(3 Pt 1):527-33.

7. Lee, C. H. et al. Pulmonary tuberculosis and delay in anti-tuberculous treatment are important risk factors for chronic obstructive pulmonary disease. PLoS One 7, e37978, 10.1371/journal.pone.0037978 (2012).

8. Airio, A., Kauppi, M., Kautiainen, H., Hakala, M. \& Kinnula, V. High association of mycobacterial infections with polymyositis in a non-endemic country for tuberculosis. Ann Rheum Dis 66, 14041405, 10.1136/ard.2007.070177 (2007).

9. Chu, $\mathrm{H}$. et al. Risk of tuberculosis among healthcare workers in an intermediate-burden country: a nationwide population study. J Infect 69, 525-532, 10.1016/j.jinf.2014.06.019 (2014).

10. Butt G, Asad F, Khurshid K et all. Frequency of pulmonary tuberculosis in patients with skin diseases requiring high dose long-term systemic steroid therapy. JPAD, 2013; 23 (2):126-132.

11. Davies PDO. Risk factors for tuberculosis. Monaldi Arch Chest Dis. 2005; 63:37-46.

\section{AUTHORSHIP AND CONTRIBUTION DECLARATION}

\begin{tabular}{|c|c|c|c|}
\hline Sr. \# & Author-s Full Name & Contribution to the paper & Author $=$ s Signature \\
\hline 1 & Dr. Humaira Talat & $\begin{array}{l}\text { Data collection \& analyzing } \\
\text { data }\end{array}$ & houed- \\
\hline 3 & $\begin{array}{l}\text { Dr. Reema Mirza } \\
\text { Dr. Madiha Sajid }\end{array}$ & $\begin{array}{l}\text { Abstract, introduction, } \\
\text { resutls, discussion } \\
\text { Conclusion \& references }\end{array}$ & fancos \\
\hline 4 & Dr. Maria Mansoor & Result analysis & Whatud \\
\hline 5 & Dr. Zarnaz Wahid & Supervising all items & DS \\
\hline 6 & Dr. Saba Majeed & Data collection & \\
\hline
\end{tabular}

